\title{
Neuroanatomy of visual acuity
}

from Peter Lennie

ONE of the aims of vision research is to find the structures that underly our visual capabilities; sometimes the relation between structure and function is striking. There is a regular correspondence between points on the retina and points on the primary visual cortex in man, but because the cortical projection of the fovea (the area of the retina responsible for the resolution of fine detail) is huge in relation to the projection of the peripheral retina, the map on the cortex is a distorted representation of the retinal surface. The large projection of the foveal region seems to be required for the resolution of fine detail, for there is a close relation between the "cortical magnification factor" $(M)$-the distance in $\mathrm{mm}$ on the visual cortex coresponding to an angle of one degree subtended at the eye-and visual acuity, expressed as the angle subtended at the eye by the smallest object that can be resolved. Cowey and Rolls (Expl Brain Res. 21, 447; 1974) showed a high correlation between $1 / M$ and the minimum angle of resolution (MAR) at different points in the visual field, which suggests that a constant amount of visual cortex is devoted to the minimum angle of resolution, whatever its value.

The much magnified cortical representation of the foveal region may originate in the distribution across the retina of the ganglion cells whose axons project to the lateral geniculate nucleus (LGN), which relays to the cortex. With the exception of the foveal region the density of ganglion cells decreases regularly with increasing distance from the fovea, and is found to be proportional to $M^{2}$. Thus over most of the visual field the magnification factor could be explained by assuming that, on average, each ganglion cell projects to an equal area of visual cortex. In the foveal region, where acuity is best and cortical magnification highest, the relationship between ganglion cell density and $M^{2}$ breaks down however, probably because the ganglion cells are arranged in a band around the foveal cones to which they are connected and thus are displaced from their receptive fields.

If the displacement of ganglion cell bodies causes the breakdown of the proportionality between ganglion cell density and $M^{2}$, an allowance for this displacement should extend the proportionality into the very centre of the fovea. Rolls and Cowey (Expl Brain Res. 10, 298; 1970) have done this for monkeys and Drasdo (this issue of Nature, page 554 has now made a correction for ganglion cell displacement in man, by computing the density of ganglion cell receptive fields at different distances from the fovea. He has shown an impressive correlation between calculated receptive field density and $M^{2}$ from the fovea to points $40^{\circ}$ in the peripheral retina, and has also formulated an equation for which he claims some generality in predicting visual performance at any point in the visual field.

These findings suggest a remarkable simplicity in the arrangement of the mechanism subserving visual acuity. However, other recent work has revealed new complexities in the structure and function of the visual system that make these simple relations rather surprising.

One of these is that retinal ganglion cells project to several areas of the brain (notably to the superior colliculus) besides the visual cortex. We cannot be clear therefore about the significance of the simple relations between visual acuity, cortical magnification and the overall density of ganglion cells until we know what fraction of cells projects to the visual cortex, and whether this fraction varies with position on the retina. The answer to this question is unclear, although recent evidence (Bunt et al. J. comp. Neurol. 164, 265; 1975) suggests that in fact virtually all ganglion cells project to the cortex through the LGN and therefore that projections to other areas arise through collateral axons from the same cells.

A more intriguing problem arises from the fact that there are several different types of ganglion cell, distinguishable histologically (and in the monkey also physiologically), and so we also need to know how the different cell types contribute to the population of cells that does project to the cortex. Although it is now more than 30 years since Polyak gave a thorough anatomical description of different cell types in the primate retina, only recently has much attention been given to their possible functional roles. The impetus to much of this work was the discovery in the cat's retina of several classes of ganglion cell that are physiologically and morphologically distinctive and in some cases at least have different projections in the brain (see Levick in Nature 254, 659; 1975 for a review). The primate visual system also seems to contain ganglion cell types that are physiologically distinctive (De Monasterio and Gouras J. Physiol. Lond. 251, $167 ; 1975)$, although these seem to be less clearly differentiated and are not so obviously related to morphology. One is therefore led to ask what different functions they subserve and whether their projections on the primary visual cortex are topographically different.

Since the physiologically different cell types are distributed differently on the retina it seems likely that they will project differently on the primary visual cortex. This being the case it is puzzling that $M$, which must reflect the projections of all classes of ganglion cell, should be so simply related to MAR, which one supposes might be mediated by a single cell type. Perhaps this class is predominant, and cells of other types, even if they differ in their distribution on the retina and their projections on the cortex, occur in numbers too small to upset the simple relation between cortical magnification and minimum angle of resolution.

We might obtain some clearer idea of the functions of the putative minor classes of cell if a variety of tasks were used to examine different forms of visual acuity in relation to position in the visual field. At all events, the heterogeneity of ganglion cells argues for caution in interpreting relations between the gross structure and function of the visual pathway.

\section{Historical ichthyology}

\section{from a Correspondent}

TAXONOMists are forced by the disciplines of their calling to be continually aware of the past history of their field of study. At a basic level they are obliged to refer to the earliest description or published name for an animal or plant, and possibly more than in most sciences the constant reference back to the early literature is part of the taxonomist's way of life; to a large extent the early literature is as significant as later syntheses. In this context it is not surprising that traditional taxonomists are of ten deeply involved with the history of their field, or with the life and work of earlier students of their subject. A recently published study of Francis Day (18291889) and his collections of Indian fishes by P. J. P. Whitehead and P. K. Talwar (Bull. Br. Mus. nat. Hist. (Hist. Ser.) 5,$1 ; 1976$ ) is an excellent example 